\title{
Letter to the Editor: "Sarcopenia and Back Muscle Degeneration as Risk Factors for Back Pain: A Comparative Study"
}

\author{
Hina Vaish \\ Maharishi Markandeshwar Institute of Physiotherapy and Rehabilitation, Maharishi Markandeshwar (Deemed to be University), Mullana, India
}

To the Editor,

The Asian Spine Journal recently published an article in volume 14(3), 2020 by Kim et al. [1] "Sarcopenia and back muscle degeneration as risk factors for back pain: a comparative study." We have read the article with great interest. The introduction is well written. Also, the author had well explained about degree of back pain association with back muscle degeneration than with sarcopenia. They developed back muscle degeneration risk index, indicating it to be a useful parameter for evaluation of back pain and muscle degeneration. We gathered valuable information and congratulate the authors for this. However, there are some issues, which needs clarification.

The sample size of the study was not determined which is needed for deriving meaning conclusion. If the sample size is less than the optimum sample size, even the most thoroughly executed study may estimate those effects or associations too imprecisely. And, if the sample size is too large, may even lead to a loss in accuracy [2]. Also, the subjects were taken from one center so, identified risk factors may be unique to that single center. This methodological choice may weaken the generalizability of the study findings [3].
The author did not mention about normality test. Though, for meaningful conclusions, assumption of the normality should be followed regardless of the sample size. Choosing the wrong representative data and calculating significance level using the value of data set might give erroneous interpretation. If appropriate, then parametric test is used to compare means, otherwise medians are used to compare the groups, using nonparametric methods [4]. SPSS provides the Kolmogorov-Smirnov test for normality test of sample size $>50$ [5].

The authors have reported that comparison of backpain severity between sarcopenia/non-sarcopenia and high/low back muscle degeneration groups was compared by Student $t$-test or Mann-Whitney $U$-test. The statement is confusing regarding use of parametric or non parametric test at the same time.

Results reported that there was greater prevalence of sarcopenia in back pain group. Type 2 diabetes mellitus is associated with sarcopenia and frailty [6]. Studies have found a link between hyperglycemia and the biochemical events that may provide a potential mechanism by which diabetes may contribute to back pain [7]. There has been no mention of history taking or interview or measurement of diabetes in the subjects. There may be a probabil-

Received Jun 25, 2020; Accepted Jun 25, 2020

Corresponding author: Hina Vaish

Maharishi Markandeshwar Institute of Physiotherapy and Rehabilitation, Maharishi Markandeshwar (Deemed to be University), Mullana, Ambala, Haryana, 133207, India

Tel: +91-9450124758, E-mail: hinavaish@mmumullana.org, hina22vaish@gmail.com 
ity that back pain patient group may have more number of diabetes subjects compared to controls contributing to muscle weakness leading to pain and sarcopenia.

Next under discussion authors report regarding reliability testing of lumbar extensor muscles on 30 subjects though there was no aim mentioned like this nor anything of such kind was mentioned under methodology. Neither under subject recruitment and methodology, authors mention about 30 more participants for reliability testing and procedure for reliability testing of lumbar extensor muscles.

The study provides valuable information but the abovementioned points need to be considered for clinical interpretation.

\section{Conflict of Interest}

No potential conflict of interest relevant to this article was reported.

\section{ORCID}

Hina Vaish: https://orcid.org/0000-0001-6045-845X

\section{References}

1. Kim WJ, Kim KJ, Song DG, et al. Sarcopenia and back muscle degeneration as risk factors for back pain: a comparative study. Asian Spine J 2020;14:36472.

2. Zodpey SP. Sample size and power analysis in medical research. Indian J Dermatol Venereol Leprol 2004;70:123-8.

3. Song JW, Chung KC. Observational studies: cohort and case-control studies. Plast Reconstr Surg 2010;126:2234-42.

4. Mishra P, Pandey CM, Singh U, Gupta A, Sahu C, Keshri A. Descriptive statistics and normality tests for statistical data. Ann Card Anaesth 2019;22:67-72.

5. Ghasemi A, Zahediasl S. Normality tests for statistical analysis: a guide for non-statisticians. Int J Endocrinol Metab 2012;10:486-9.

6. Umegaki H. Sarcopenia and diabetes: hyperglycemia is a risk factor for age-associated muscle mass and functional reduction. J Diabetes Investig 2015;6:6234.

7. Rinaldo L, McCutcheon BA, Gilder H, et al. Diabetes and back pain: markers of diabetes disease progression are associated with chronic back pain. Clin Diabetes 2017;35:126-31. 